\title{
Force Evolutionary Billiards and Billiard Equivalence of the Euler and Lagrange Cases
}

\author{
V. V. Vedyushkina ${ }^{a, *}$ and Academician of the RAS A. T. Fomenko ${ }^{a, * *}$ \\ Received January 23, 2021; revised January 23, 2021; accepted January 26, 2021
}

\begin{abstract}
A class of force evolutionary billiards is discovered that realizes important integrable Hamiltonian systems on all regular isoenergy 3-surfaces simultaneously, i.e., on the phase 4-space. It is proved that the well-known Euler and Lagrange integrable systems are billiard equivalent, although the degrees of their integrals are different (two and one).
\end{abstract}

Keywords: integrable system, billiard, billiard book, Liouville equivalence, Fomenko-Zieschang invariant, evolutionary force billiards, rigid body dynamics

DOI: $10.1134 / \mathrm{S} 1064562421010154$

The following general question has arisen repeatedly: can an integrable Hamiltonian system (IHS) with two degrees of freedom be realized by a single billiard system on the entire phase 4-manifold $M^{4}$ ?

It is well known that many IHS in mechanics, physics, and their analogues on Lie algebras were previously characterized by a "code," namely, by FomenkoZieschang invariants [1] of Liouville foliations of a system with constraints imposed on the set of isoenergy $3-$ surfaces $Q^{3}$ (arranged in increasing order of the energy $H$ ) which represent regular energy zones of the system (see [1-3]).

Such systems were then realized using several billiards (see [4-6]): for a regular energy zone of a system (for any of its $Q^{3}$ ) its own billiard with the same Fomenko-Zieschang invariant was constructed. For example, for geodesic flows of Riemannian metrics (e.g., on conics [7]) and for billiards introduced by Vedyushkina on CW complexes (billiard books [8] and topological billiards [9]), the particle's energy $H=|V|^{2}$ is only a scaling parameter. It turns out that these classes are important in applications.

When a potential is added to a billiard or geodesic flow on a 2-surface, the dependence of the foliation topology on energy becomes nontrivial and changes occur not only in the code of the system, but also in its other, purely four-dimensional invariants, including the topology of its 4-singularities. Many IHS contain 4-singularities, both nondegenerate (classified by N.T. Zung,

\footnotetext{
${ }^{a}$ Faculty of Mechanics and Mathematics, Lomonosov Moscow State University, Moscow, 119991 Russia

*e-mail: arinir@yandex.ru.

**e-mail: atfomenko@mail.ru
}

see [1]) and degenerate (see [10] for parabolic singularities). The study of them is a another difficult subject. Invariants of systems with potentials were investigated in [11-13]. At the same time, adding a potential, for example, to a locally Euclidean billiard complicates the trajectories of the material particle, namely, they cease to be geodesics of flat metrics. In contrast, we want to realize IHS by billiards with classical motion of particles over geodesics of locally flat metrics.

The task is to find a class of locally flat billiards suitable for realizing the code of an IHS, i.e., the topology of its Liouville foliations (closures of its trajectories) in all regular energy zones without introducing potentials or imposing additional conditions on the four-dimensional topology of the system.

The answer turned out to be positive: Fomenko proposed a new class of billiards, namely, force (or evolutionary) billiards. In them, both the topology of the billiard table and the law of ball reflection can change by varying the velocity of the ball (the "force" of the impact on the wall boundary). The following definitions were introduced by Fomenko.

Definition 1. The support of a force billiard is a finite connected two-dimensional locally flat (with a Euclidean metric inside 2-cells) CW complex $X$. Its 2 -sheets $L_{i}$ are homeomorphic to closed simply connected domains $R^{2}$ and are bounded by a piecewise smooth curve. Its angles at junction points are equal to $\pi / 2$. Several 2-sheets are glued along an isometry of their common smooth boundary arc (book spine).

Definition 2. For each value of theparameter (energy) $H=h \geq 0$, a closed possibly nonconnected subcomplex $X(h)$ of the support $X$ is called the state of the force billiard corresponding to the energy value $h$. 


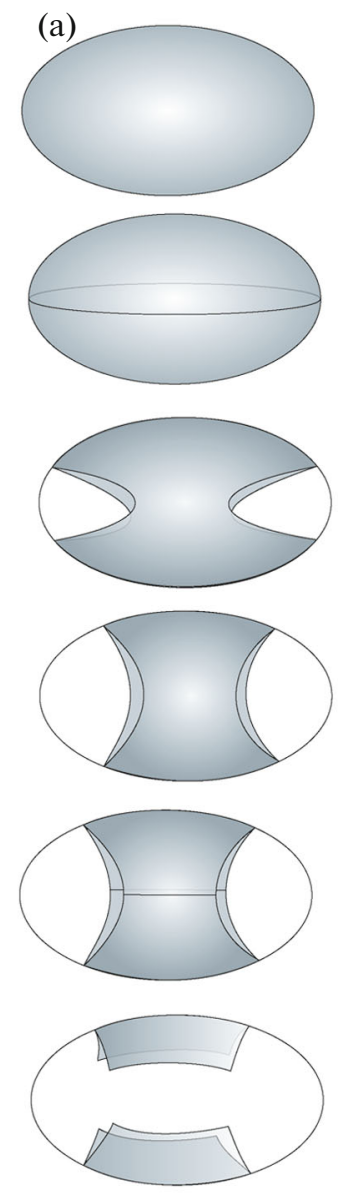

(b)

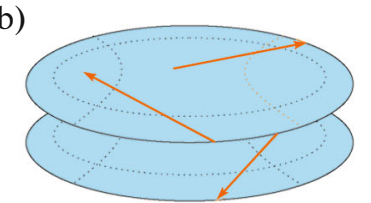

\begin{tabular}{l|l}
$g$ & $g=$ const
\end{tabular}
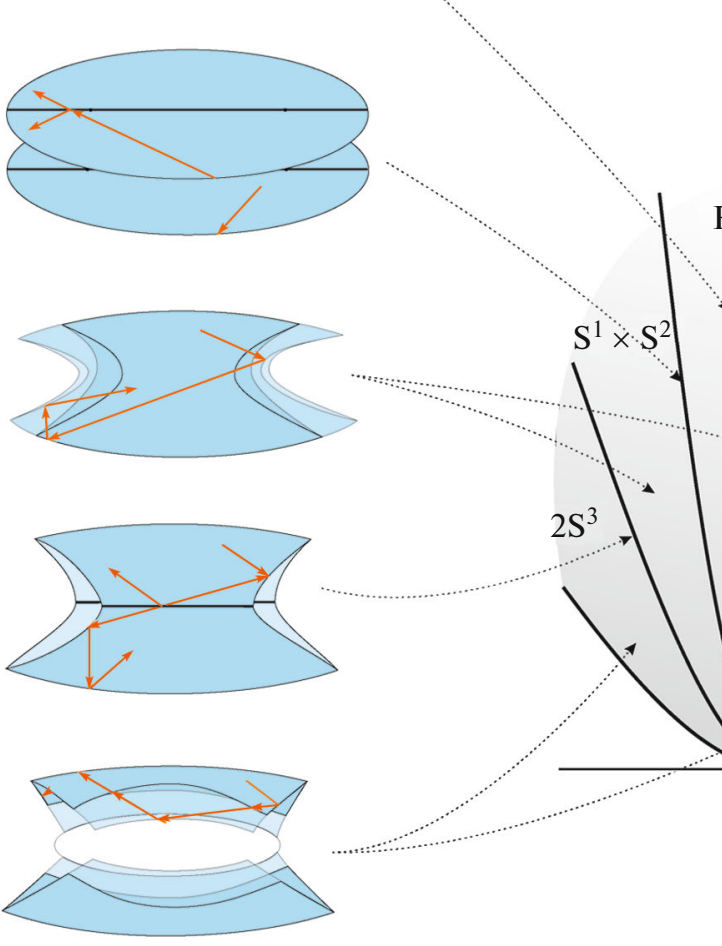

\section{$\vec{g}$}

Fig. 1. Realization of the Euler case of force billiards: the states of the force billiard shown as (a) subsets of an ellipsoid and as (b) plane domains, and the bifurcation diagram of the area integral and the energy of the Euler system.

Here, $X\left(h_{1}\right) \subseteq X\left(h_{2}\right)$ for any $h_{1}<h_{2}$ and $X=\cup X(h)$ over all $h$. Therefore, the state $X(h)$ "enlarges" with increasing $h$. In Fig. 1a, the states of a force billiard are shown as subsets of an ellipsoid $E^{2}$.

Definition 3. The finite set of values $h=1, \ldots, N$ of the energy $H$ for which the table topology or the reflection/refraction law on boundary edges changes are called singular, while the other energy values are called regular. Recall that 1-edges (spines) of a billiard $X(h)$ are arcs of confocal quadrics or concentric circles. In Fig. 1b, singular energy values 1 and 2 correspond to two transitions (between states).

Definition 4. The law of reflection/refraction on an edge (spine) $r$ at a state $X(h)$ is denoted by $Z(h, r)$. It is specified by a cyclic permutation on $n$ sheets glued along the edge $r$ and determines the dynamics of the particle after the impact on the boundary. Suppose that a set of such laws $Z(h)=\{Z(h, r)\}$ is a piecewise constant function of energy with changes occurring only at singular values $h$.

Definition 5. Edge spines of a state $X(h)$ are allowed to vary smoothly in the class of confocal quadrics without degenerations. As a result, according to the theory of integrable billiards [14], equivalent billiards are defined [4]. For singular $h$, edges can be glued to other edges, can degenerate, or can transform into segments of focal lines. Sheets are glued along their boundary arcs that belong to the same quadric. On a "new spine," there appears a new cyclic permutation. At the moment of a jump, the equivalence class of billiards is allowed to change. For example, for singular $h$, a boundary segment can get onto a focal line or can jackknife. An angle of $\pi / 2$ can change in a jump into $\pi$. Spines of a state $X(h)$ are allowed to be glued at boundary points if they lie on a single boundary arc, i.e., if the angle between them is equal to $\pi$. In circular billiards, boundary circles can contract to points.

For example, in Fig. 1b, for each of two singular values $h$, the corresponding edge $r$ becomes "permeable" ("transparent"), so that the billiard ball passes through it, while having reflected from it previously. Moreover, the pair of identity permutations on elliptic arcs is replaced by transpositions on a focal line segment.

Definition 6. The support $X$ is assumed to be "stationary." The states $X(h)$ in it enlarge, and $X$ coincides 


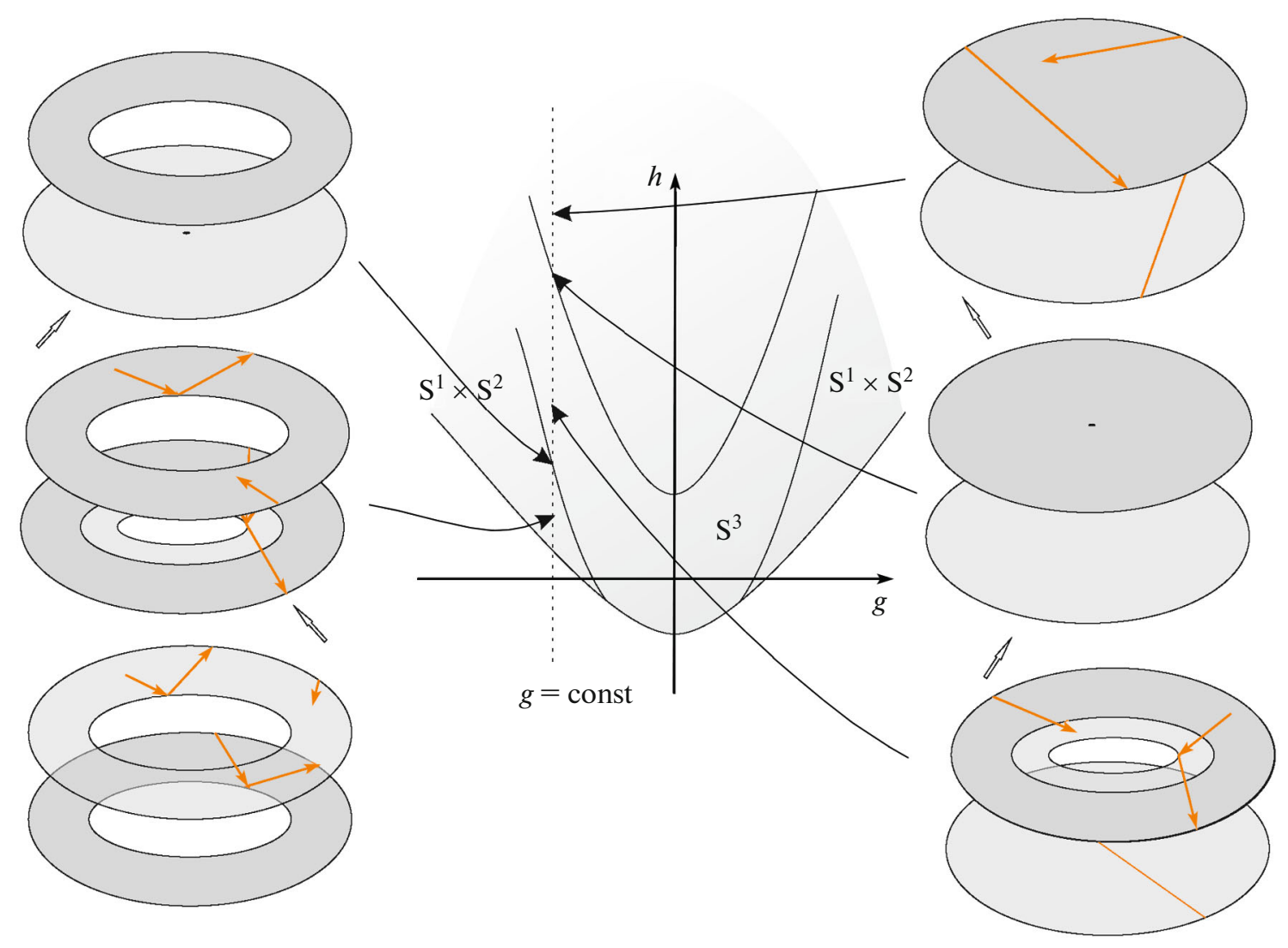

Fig. 2. Force billiard realizes a symplectic leaf of the Lagrange system.

with the last state $X(N)$. An integrable system with two degrees of freedom defined by the dynamics of the billiard ball on the changing states $X(h)$ is called a force (evolutionary) billiard.

Let $h$ be a regular energy value from a certain interval $D_{i}=(i, i+1)$. The corresponding billiard state is denoted by $X\left(D_{i}\right)$.

Definition 7. A point of the phase complex $T X\left(D_{i}\right)$ is a pair $(x, v)$, where $x$ is a point of the billiard table $X\left(D_{i}\right)$ and $v$ is the velocity of the material particle at the point $x$. When the point $x$ reaches the boundary of a sheet $L_{i}$ neighboring a sheet $L_{k}$, the corresponding pairs $(x, v)$ and $(x, w)$ are glued together according to the reflection/refraction law $Z(h, r)$ acting on the given edge $r$ for the given $h$.

Definition 8. A regular isoenergy 3-surface $Q_{h}$ is a subset of the four-dimensional phase complex $T X\left(D_{i}\right)$ defined by the equation $H=h$, i.e., this is a "level of constant energy." For integrable billiard books, regular $Q_{h}$ are topological 3-manifolds [15].

Consider the famous Euler case (1750) in the dynamics of a heavy rigid body fixed at the center of mass [1]. It has a first integral that is quadratic with respect to the momentum components. The topology of a symplectic leaf $M_{g}^{4}$ of the system does not depend on the choice of a nonzero value $g$ of the area integral $f_{2}$. In the rightmost panel in Fig. $1 \mathrm{~b}, M_{g}^{4}$ is associated with the preimage of the vertical line under the mapping $\left(f_{2}, H\right)$. The singular isoenergy surfaces $Q$ are mapped to points of three parabolas. Each 2-domain is assigned a homeomorphicity class of the preimage of any of its points-a regular $Q_{g, h}^{3}$.

Let us construct a force billiard for such a leaf $M_{g}^{4}$ (see Fig. 1). Its support is a gluing of two domains bounded by an ellipse and is homeomorphic to a 2ellipsoid $E^{2}$. In Fig. 1a, the states of the billiard are shown as subsets of $E^{2}$, while, in Fig. 1b, they are shown as gluing of plane domains. States with lower energy are shown lower, and vice versa. The initial (starting) state is a nonconnected billiard having no common points with the focal line (bottom). It is homeomorphic to two disks and realizes the Euler system on a pair of 3 -spheres $2 \mathrm{~S}^{3}$. Then it transforms into an annulus realizing a product of a 2 -sphere and a circle: $S^{1} \times S^{2}$. Next, the annulus turns into a sphere (ellipsoid) and realizes a projective space $\mathrm{RP}^{3}$. Figure $1 \mathrm{~b}$ also shows the trajectories of the billiard ball and the gluing of spines. The integrability of the billiard at every time of its evolution follows from the fact that its walls belong to arcs of confocal quadrics. 
Theorem 1. The constructed integrable force billiard with a support homeomorphic to an ellipsoid realizes (in the sense of Liouville equivalence) the integrable Euler case on the entire phase manifold $M_{g}^{4}$, i.e., on all of its regular isoenergy 3-surfaces for all regular values $g$ and $h$.

The Lagrange case, which admits a linear integral, describes the motion of an axisymmetric heavy rigid body with a point fixed on the axis of symmetry. It is well known that, depending on the parameter values, there exist bifurcation diagrams of four types (see [1]). In this case, it was found that there are exactly five different types of symplectic 4-leaves. For all of them, we discovered force billiards. One of them is shown in Fig. 2.

Theorem 2. On each regular symplectic 4-leaf $M_{g}^{4}$, the Lagrange integrable case is realized (in the sense of Liouville equivalence) by one of five constructed force billiards, whose billiards states are bounded by concentric circles (and, hence, are integrable at every moment of the evolution).

Let us deform the family of confocal ellipses and hyperbolas into a family of concentric circles and radial rays (by sending the foci to each other).

Theorem 3. Under the described deformation of the confocal billiards into circular ones, the force billiard realizing the Euler case is mapped to a new force billiard such that its complete set of Liouville foliations coincides with the complete set of Liouville foliations of the Lagrange case (for all three types of isoenergy 3-surfaces). Such systems are called billiard-equivalent.

Under the found transformation of the Euler case into the Lagrange one, a symplectic leaf of the Euler case is not mapped to any of the five types of symplectic leaves of the Lagrange case. Instead, this transformation has a more complex structure. This feature prevented one from observing the transformation of these systems into each other earlier. Nevertheless, it turned out that the complete set of Liouville foliations of the Euler case transforms into the complete set of Liouville foliations of the Lagrange case. First, we had to find "hidden confocal quadrics" in the Euler case and "hidden concentric circles" in the Lagrange case. Eventually, it is the deformation of confocal quadrics into circles (when the foci merge) that turns the Euler case into the Lagrange one.

Additionally, we found force billiards realizing the integrable Goryachev-Chaplygin-Sretensky case simultaneously in several regular energy zones.

\section{FUNDING}

This work was supported by the Russian Foundation for Basic Research, project no. 19-01-00775-a.

\section{OPEN ACCESS}

This article is distributed under the terms of the Creative Commons Attribution 4.0. International Public License (http://creativecommons.org/licenses/by/4.0/), which permits unrestricted use, distribution, and reproduction in any medium provided you give appropriate credit to the original author(s) and the source, provide a link to the Creative Commons license, and indicate if changes were made.

\section{REFERENCES}

1. A. V. Bolsinov and A. T. Fomenko, Integrable Hamiltonian Systems: Geometry, Topology, and Classification (Udmurt. Univ., Izhevsk, 1999), Vol. 1.

2. V. A. Kibkalo, Sb. Math. 210 (5), 625-662 (2019).

3. V. Kibkalo, Topol. Appl. 275, 107028 (2020).

4. V. V. Vedyushkina (Fokicheva) and A. T. Fomenko, Izv. Math. 81 (4), 688-733 (2017).

5. V. V. Vedyushkina and A. T. Fomenko, Dokl. Math. 486 (2), 151-155 (2019).

6. V. V. Vedyushkina (Fokicheva) and A. T. Fomenko, Izv. Math. 83 (6), 1137-1173 (2019).

7. G. V. Belozerov, Sb. Math. 211 (11), 1503-1538 (2020).

8. V. V. Vedyushkina and I. S. Kharcheva, Sb. Math. 209 (12), 1690-1727 (2018).

9. V. V. Vedyushkina, Sb. Math. 210 (3), 310-363 (2019).

10. A. Bolsinov, L. Guglielmi, and E. Kudryavtseva, Phil. Trans. R. Soc. A 376, 20170424 (2018).

11. I. F. Kobtsev, Moscow Univ. Math. Bull. 73 (2), 64-70 (2018).

12. I. F. Kobtsev, Sb. Math. 211 (7), 987-1013 (2020).

13. S. E. Pustovoitov, Fundam. Prikl. Mat. 22 (6), 201-225 (2019).

14. V. V. Kozlov and D. V. Treshchev, Billiards: A Genetic Introduction to the Dynamics of Systems with Impacts (Mosk. Gos. Univ., Moscow, 1991; Am. Math. Soc., Providence, R.I., 1991).

15. I. S. Kharcheva, Moscow Univ. Math. Bull. 75 (4), 149-160 (2020).

Translated by I. Ruzanova

DOKLADY MATHEMATICS Vol. 103 No. 1 2021 